\title{
Fast and Broadband Fiber Dispersion Measurement with Dense Wavelength Sampling
}

\author{
Giorgio M. Ponzo ${ }^{(1)}$, Marco N. Petrovich ${ }^{(1)}$, Xian Feng ${ }^{(1)}$, Peter Horak ${ }^{(1)}$, Francesco Poletti ${ }^{(1)}$, \\ Periklis Petropoulos ${ }^{(1)}$ and David J. Richardson ${ }^{(1)}$ \\ (1) Optoelectronics Research Centre, University of Southampton, UK, mnp@orc.soton.ac.uk
}

\begin{abstract}
We report on a method to obtain accurate dispersion measurements from low-coherence interferograms. This novel phase extraction method enables high accuracy, broadband measurements and very dense (20points/nm over 500nm) datasets for both dispersion and dispersion slope.
\end{abstract}

\section{Introduction}

The accurate measurement of the group velocity dispersion (GVD) of optical fibers over broad wavelength intervals (several hundred $\mathrm{nm}$ ) is key to the efficient exploitation of nonlinear optical effects. For instance, it is essential for optimizing the shape and spectral extent of supercontinuum generation ${ }^{1}$, or to ensure phase matching conditions in parametric processes ${ }^{2}$, or to control effects such as pulse compression and soliton self-frequency shift ${ }^{3}$. It is also desirable to obtain dense GVD datasets (i.e. closely spaced data points) to enable accurate determination of the higher dispersion orders, which are critical e.g. for applications involving pulse compression ${ }^{4}$ and soliton generation ${ }^{5}$. As the above mentioned applications typically require short (a few meters) device lengths, the ability to measure short samples is of great value. Microstructured optical fibers (MOFs), incorporating arrays of wavelength-scale air holes (or other high-index-contrast features) afford large flexibility in designing the fiber dispersion. However, often the GVD is very sensitive to the fiber's structural parameters and thus a dispersion measurement technique that can provide high accuracy over broad wavelength intervals is paramount for both fiber development and application work.

Dispersion measurements in short fiber samples are commonly based on low-coherence interferometry $(\mathrm{LCl})$. Here, interferograms are produced by combining two broadband beams, one directed through the fiber under test, and the other directed through a free-space variable delay line. The interference can be detected either in the temporal ${ }^{6}$ or in the spectral domain $^{7}$. In the spectral domain, the wavelength dependent GVD can in principle be extracted from single-shot interferograms, which can extend over intervals as wide as a few hundred $\mathrm{nm}$. To retrieve the phase from the wavelength dependent intensity, two methods are widely used. The first consists in finding the intensity maxima and minima, which correspond to a $2 \pi$ phase shift ${ }^{8}$. This straightforward approach has however the drawback of significantly underexploiting the information contained in the interferogram as it only outputs a small set of GVD values and thus cannot yield reliable information on the dispersion slope. In contrast, a direct nonlinear $\mathrm{fit}^{7}$ of the interferogram can in principle yield denser datasets, however this approach requires the initial conditions to be set on a case-by-case basis and verification of the quality of the fit, which is very time consuming and often leads to sub-optimal results.

In this work, we present a novel LCl method based on a direct phase extraction scheme, which achieves an unprecedented combination of broad bandwidth, dense wavelength sampling intervals, high accuracy and a fast measurement capability. To illustrate its versatility, we apply it to the case of three microstructured fiber types with widely ranging dispersion profiles.

Interferometric setup, method and validation The setup for the dispersion measurement (Fig.1a) uses a broadband SC source (450$2200 \mathrm{~nm})$ and a free-space Mach-Zehnder interferometer incorporating polarization control and motorized shutters in each arm. The optical elements used in our setup were carefully chosen to provide the widest broadband operation. The normalized intensity, $I_{N}$, at the

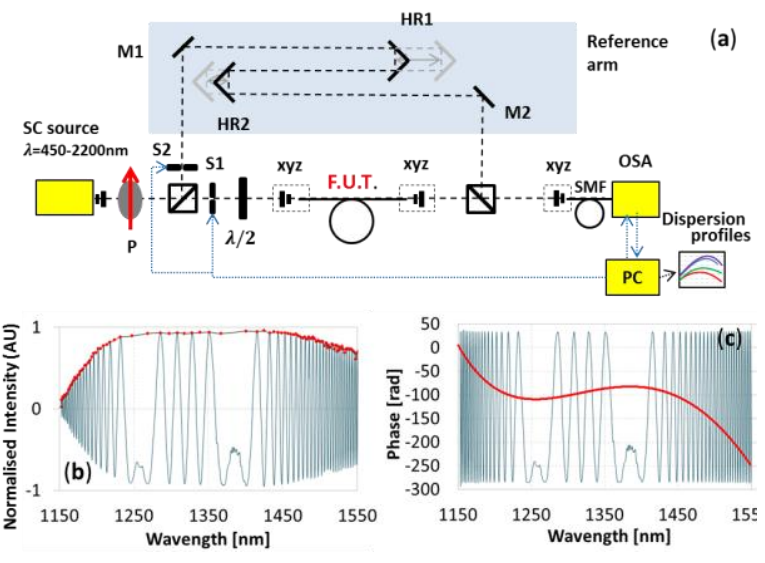

Fig. 1: (a) Schematic of setup (b) Interferogram and envelope function $\mathrm{V}(\lambda)$ (c) Interferogram divided by the visibility and reconstructed phase. 
OSA (shown in Fig.1b) can be expressed as:

$$
I_{N}(\lambda)=\frac{I(\lambda)-I_{R}(\lambda)-I_{F}(\lambda)}{2 \sqrt{I_{R}(\lambda) I_{M}(\lambda)}}=V(\lambda) \cdot \cos \varphi(\lambda)
$$

$I_{R}, I_{F}, I$ are the reference, measurement and combined beam intensities, $\varphi=\beta L-2 \pi / \lambda d$ is the phase and $V(\lambda) \leq 1$ is the envelope or visibility function that defines the width of the interferogram (Fig.1b). To extract the phase, $\mathrm{V}(\lambda)$ is first reconstructed by linear interpolation of the intensity maxima/minima as shown in Fig.1b. The normalized intensity is then divided by the envelope, and the phase is obtained as:

$$
\varphi(\lambda)=\arccos \left[\frac{I_{N}(\lambda)}{V(\lambda)}\right]
$$

In solving Eq. 2, the $2 \pi$ ambiguity of the arccos function is resolved by considering that a $2 \pi$ phase shift exists between two subsequent maxima or minima. More importantly, we define a 'fringe spacing function' and search for its local maxima (i.e. the 'centers of symmetry' (CoS) of the interferogram) to account for the phase sign inversion occurring at these points (see Fig. 1c). The GVD is finally determined as:

$$
D(\lambda)=-\frac{1}{2 \pi c L}\left[2 \lambda \frac{d \phi}{d \lambda}+\lambda^{2} \frac{d^{2} \phi}{d \lambda^{2}}\right]
$$

By using Eq. 2-3, very dense datasets (up to 20 points $/ \mathrm{nm}$ ) can be easily obtained and, as opposed to fit methods, calculation is very fast.

For a given OSA resolution, the spectral extent of the interferogram is maximized if the interferogram is resolved in proximity of its center of symmetry, as per Fig.1c. In contrast to the maxima/minima method described in the introduction, reliable analysis in the vicinity of the CoSs is possible with the proposed method.

Systematic errors may arise due to any differential dispersion between the arms and require correction, particularly in the case of fibres with flat, near-zero dispersion. The small contributions due to the wave-plate and objectives were determined from the phase difference of the interferograms of a standard single mode fiber. Another and potentially more serious issue may arise from environmental perturbations causing time-varying changes in the relative phase along the two paths, which we sought to minimize by placing the setup in an enclosure. Whilst for an un-isolated system a measurable error of $>2 \mathrm{ps} / \mathrm{nm} / \mathrm{km}$ was observed even for a relatively short scan time of $\sim 60$ s, for an isolated setup $<0.1 \mathrm{ps} / \mathrm{nm} / \mathrm{km}$ variations were observed for scan times as long as 360 s.

The overall accuracy of the present method was estimated by comparing the dispersion measured for a standard SMF with theoretical predictions based on experimental refractive index profiles. The result (Fig. 2a) shows excellent agreement with $<0.5 \mathrm{ps} / \mathrm{nm} / \mathrm{km}$

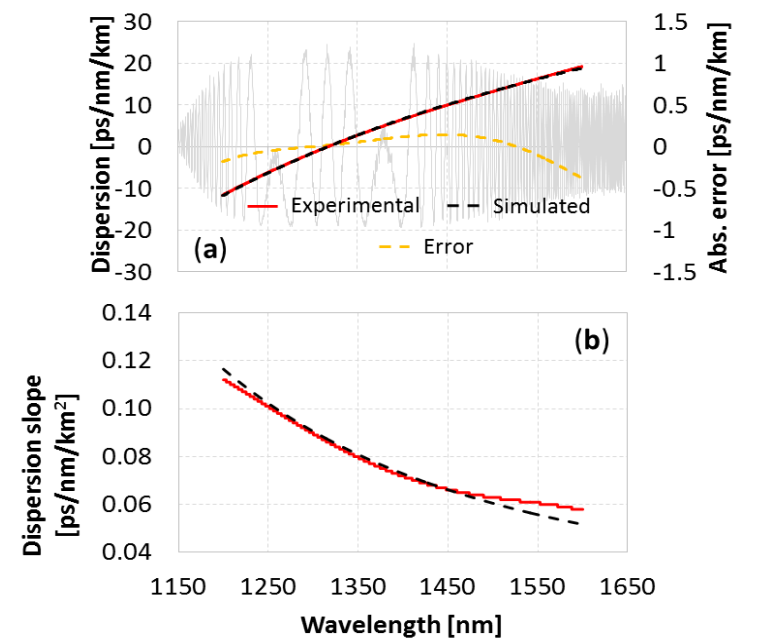

Fig. 2: Modeled and experimental dispersion (a) and dispersion slope (b) profiles of a standard SMF illustrating the accuracy of method.

difference over at least 400nm (1200-1600nm). To illustrate the importance of dense wavelength sampling, the dispersion slope has also been calculated and compared (Fig. 2b). Excellent agreement is equally obtained for the dispersion slope over most of the $400 \mathrm{~nm}$ wide interval.

\section{GVD measurement of key MOF fiber types}

The technique was applied to measure the GVD profiles of three microstructured fibers with very different dispersion properties.

The first is an all-solid MOF based on three different lead-silicate glasses arranged to produce a $\mathrm{W}$-type index profile ${ }^{2}$. This ' $\mathrm{W}$-fiber' (Fig. 3b-inset) was designed to have high nonlinearity and flat, near-zero dispersion at $1550 \mathrm{~nm}$. To accurately match the design target (dispersion below a few $\mathrm{ps} / \mathrm{nm} / \mathrm{km}$ in the Cband), $W$-fibers with a few percent difference in core diameter were fabricated. To resolve the small differences in GVD, ps $/ \mathrm{nm} / \mathrm{km}$ accuracy was required. Fig. $3(a, b)$ shows the interferograms and the corresponding dispersion profiles obtained for two W-fibers with slightly different values of the core diameter, $d$. As expected, the fibers show flat and near-zero dispersion with differences as small as a few $\mathrm{ps} / \mathrm{nm} / \mathrm{km}$ between the two curves. We conclude that our technique can resolve very small differences in the dispersion profiles as required. We then tested a 7 cell hollow core photonic bandgap fiber (PBGF) also designed for $\sim 1550$ nm operation (SEM image shown in Fig. 3(c)-inset). PBGFs have dispersion profiles diverging at both the short and long wavelength bandgap edges. This enables us to further demonstrate the performance of the present technique at high values of both dispersion and dispersion slope and to estimate the 

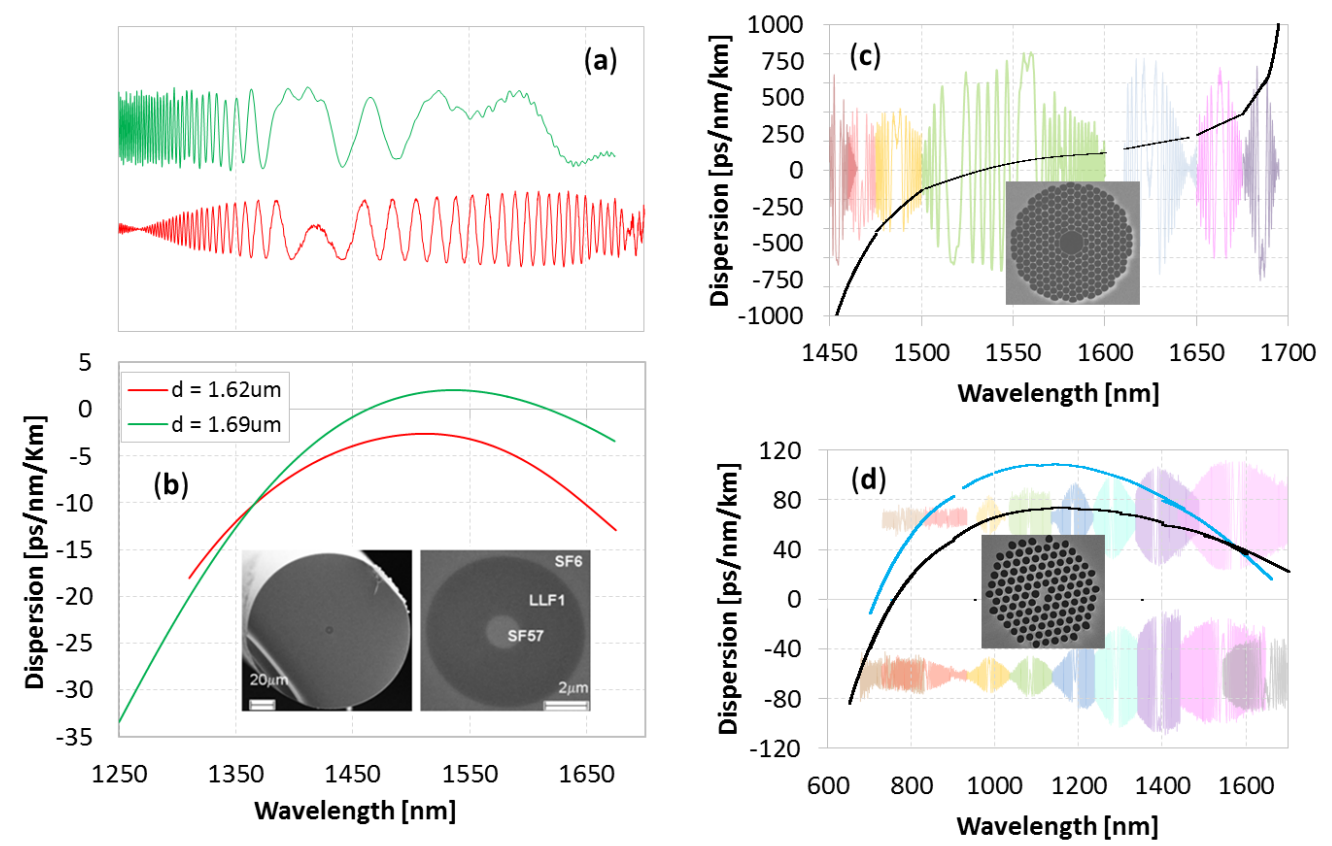

Fig. 3: (a) Interferograms and (b) GVD of two W-type MOFs; (c) measured GVD of a 7 cell PBGF; (d) polarization resolved GVD profiles of a highly birefringent high-nonlinearity MOF. Insets show SEM images.

highest/lowest measurable GVD. When the fiber dispersion is high, the interferograms have closely spaced fringes and become narrow in width due to the OSA resolution. We found that $\sim 7$ adjacent interferograms (collected by varying the length of the reference arm) were typically required to obtain the GVD over the whole bandgap ( 250 $\mathrm{nm}$ in width). The full dispersion profile could then be obtained as a collection of neighboring curves (Fig. 3(c)). The highest measurable dispersion, in this case about $\pm 1000 \mathrm{ps} / \mathrm{nm} / \mathrm{km}$, is determined by the resolution of the OSA. It should be noted that 7 cell PBGFs are slightly multimode and indeed several secondary interferograms, produced by higher order core modes, were observed. These could be minimized (but not eliminated) by optimizing the input coupling, and produced a slightly higher level of noise in the interferogram of the fundamental mode. Despite this, the curves obtained from adjacent interferograms were remarkably consistent (within $\sim 5 \%$ ).

Last, we measured a highly birefringent, high nonlinearity index-guiding MOF incorporating a strongly asymmetric core (SEM in Fig.3(d)inset). By adjusting the input half-wave plate, we obtained polarization-resolved measurements. As this fiber has moderate GVD values $(<100$ $\mathrm{ps} / \mathrm{nm} / \mathrm{km}$ ), it was possible to obtain data over a $\sim 1000 \mathrm{~nm}$ wide interval $(700-1700 \mathrm{~nm})$ by analyzing a set of 8 contiguous interferograms per polarization. The corresponding GVD curves plotted in Fig. 3(d) are again remarkably consistent. Note that the measurement range is limited by the bandwidth of the components and the OSA range only.

\section{Conclusions}

We have reported a dispersion measurement method that provides for fast $(<10 s)$, high accuracy $(<0.5 \mathrm{ps} / \mathrm{nm} / \mathrm{km})$ and absolute precision $(<0.1 \mathrm{ps} / \mathrm{nm} / \mathrm{km})$ measurements over broad spectral ranges of $>400 \mathrm{~nm}$. We have successfully applied our technique to measure microstructured fibers having markedly different dispersion profiles. The technique is ideally suited to resolve differences arising due to very small ( tens of $\mathrm{nm}$ ) structural variations and can cope with dispersion profiles having extreme curvature or dispersion values as high as $1000 \mathrm{ps} / \mathrm{nm} / \mathrm{km}$. For fibers with more moderate values of GVD ( 100ps/nm/km), measurements over intervals exceeding $1000 \mathrm{~nm}$ are possible by collecting multiple adjacent interferograms.

\section{Acknowledgements}

This work was supported by the UK EPSRC through grant EP/H02607X/1 and EP/I01196X/1.

\section{References}

[1] A.M. Heidt, J Opt Soc Am B 27, 550 (2010).

[2] A. Camerlingo, et al. Opt Express 18, 15747 (2010).

[3] J.P. Gordon, Opt Lett 11, 662 (1986).

[4] V.I. Kruglov, et al., Phys Rev A 84, 023823 (2011).

[5] M. Taki, et al. Phys Lett A 374, 691 (2010).

[6] P. Francois et al., Electron Lett 23,357 (1987)

[7] P. Merritt et al., J Lightwave Technol 7, 703 (1989).

[8] J. Jasapara, et al., J Opt Soc Am B 20, 1611 (2003). 